Research Article

\title{
Investigating the Contact Responses of the Roller Cavity Surfaces in the Compressor Blade Rolling Process
}

\author{
Qichao Jin $\mathbb{D}^{1},{ }^{1}$ Wenhu Wang, ${ }^{2}$ and Ruisong Jiang ${ }^{3}$ \\ ${ }^{1}$ Key Laboratory of Road Construction Technology and Equipment of MOE, Chang'an University, Xi'an 710064, China \\ ${ }^{2}$ School of Mechanical Engineering, Northwestern Polytechnical University, Xi'an 710072, China \\ ${ }^{3}$ School of Aeronautics and Astronautics, Sichuan University, Chengdu 610065, China \\ Correspondence should be addressed to Qichao Jin; jinqichao@chd.edu.cn
}

Received 29 April 2020; Revised 26 May 2020; Accepted 28 May 2020; Published 30 June 2020

Guest Editor: Guian Qian

Copyright (C) 2020 Qichao Jin et al. This is an open access article distributed under the Creative Commons Attribution License, which permits unrestricted use, distribution, and reproduction in any medium, provided the original work is properly cited.

\begin{abstract}
The investigation of the contact responses is the key for evaluating the local wear of dies in the plastic forming process. This paper investigated the contact load distributions and evolutions of the roller cavities in the compressor blade rolling process by the FEM. It was the first study to quantify the distributions and evolutions of the contact responses for rolling irregular components. The results indicated that the maximum contact pressure is generally present at the center of the contact interfaces, and the magnitudes of contact pressure decreased with evolution of the blade rolling process. The rolling contact interfaces can be divided into the backward slip zone, the stick zone, and the forward slip zone based on the shear stress distributions. The stick zone was a narrow belt which separated the forward and the backward slip zone, and the shear stress in the stick zone was nearly zero. The shear stress magnitudes in the forward slip zone were smaller than those in the backward slip zone, and the directions of shear stress in forward and backward slip zones were adverse. The magnitudes of shear stress over the forward and backward slip zones decreased with evolution of the blade rolling process. The distributions of local sliding were in a V-shape, the local sliding in the stick zone was nearly zero, and the bigger sliding in backward and forward slip zones was present at the boundaries of rolling entrance and exit sections. The local sliding velocity magnitudes in the backward slip zones were always bigger than those in the forward slip zones, and the magnitudes of local sliding at the rolling entrance sections were bigger than those at the rolling exit sections. In general, the local sliding velocity magnitudes increased firstly and decreased sharply at $2 T / 3$. The current paper develops the distributions and evolutions of contact responses in the blade rolling process. The contact responses can be used for studying the wear of roller cavities to avoid the accuracy inconsistency of the shaped blade.
\end{abstract}

\section{Introduction}

In the industry of the cold rolling compressor blade, the cavity surfaces of the roller undergo cyclic resistance of deformation which induces unknown wear [1]. The manufacturing accuracy is sensitive to the shape of roller cavity surfaces in compression moulding formation; this feature is particularly obvious for the net-shaped rolling compressor blade [2] because good uniformity of the netshaped blades required a pair of roller cavities with stable geometry profiles and persistent mechanical performance. However, the unknown wear of the cavity surface induced confusion of the formed blade's precision and the roller's severe life [3]. Hence, understanding of the local contact responses is the first step to study the wear of the roller cavity surfaces $[4,5]$.

The contact responses depend on many factors such as the shapes, the material property, and the relative motion of the contact bodies [6]. In previous studies, three kinds of methods including analytic, experimental, and numerical methods have been used for studying the contact responses of different kinds of dies. Jobin et al. [7] presented pressure distribution of the interface for arbitrary profile. Due to its ability to analyze arbitrary-shaped contacts, it can be a powerful design tool for evaluating contact response due to different shapes and sizes of the indenter. Chu et al. [8] proposed a mathematical model to determine the strain and stress of the tube wall in tube hydroforging and predicted the 
distribution of contact stress at the tube cross section. WeiszPatrault et al. [9-11] developed the analytical inverse method to evaluate the contact stress of the roll bite in the rolling process, and three-dimensional contact stress was computed. They also used roll-gap sensors to measure contact pressure with very short computation times and investigated the effect of parameters on the contact pressure. Lee et al. [12] examined the influence of geometrical parameters on the surface stress in the double cup extrusion process. Walbran et al. [13] developed a generic liquid composite moulding simulation to predict clamping force and stress distributions acting on moulds. Sedighi and Mahmoodi $[14,15]$ investigated the rolling contact pressure distribution on the blade, and the results showed that it distributed as a saddle shape. Yan et al. [16] used FEM to study the wear of the die radius in sheet metal stamping; the contact pressure and sliding distance were determined to calculate the wear based on wear theory. Pereira et al. [17-19] studied the evolution of contact pressure and sliding distance of the die radius in sheet metal stamping and the quantified wear of the radius in the stamping process. Jurkiewicz et al. [20] investigated the contact between two rollers of a printing using analytical, numerical, and experimental methods and proposed an analytical-empirical equation of the distance between loaded and contact zone width.

The localized contact condition and its evaluation are significant to understand the contact responses and study the wear of the joined pieces. Due to the irregular shape of the blade and the corresponding cavity surfaces, the local contact conditions of the cavity surfaces were time and location responses. The finite element method provided a practical method for detecting the local contact pressure of the cavity surfaces in the blade rolling process. This paper investigated the localized contact responses and theirevolutions over roller cavity surfaces by FEM. The distributions of contact pressure, shear stress, and local sliding were presented, respectively, and their evolutions were also enumerated, respectively.

\section{The Numerical Model of Blade Rolling Processes}

2.1. The Setup of the Numerical Model for the Blade Rolling Process. In the blade rolling process, the roller cavities suffer cyclic flow stress caused by the plastic deformation resistance of the blank. Figure 1 shows a typical compressor blade and a schematic of the blade rolling process. In order to determine the distribution and evolution of the contact responses in the blade rolling process, a FEM simulation was implemented in this paper.

The rolling process was replicated in the numerical simulation using a nonlinear Explicit FE code (ABAQUS/ Dynamic, Explicit 6.14-1). In order to analyze the contact between the cavity surface and the blank in detail, the die mesh and the blank mesh were significantly refined in the region of the respective interfaces. The top and bottom rollers were propped up by a pair of simplified axles which were treated as rigid parts, the contact interfaces were tied together, and the axles drove rollers to rotate. The interactions between the blank and the roller surface were defined using the default 'master-slave' algorithm in ABAQUS [17]. The normal and tangential contact behaves were introduced between roller and blank. The Coulomb friction model was used between the roller cavities and blank, and the friction coefficient was set as 0.29. The FEM model of the blade rolling process is shown in Figure 2. The isotropic material properties of the blank and rollers are summarized in Table 1. The plastic property of the blank was tested by the tensile experiment at room temperature, the INSTRON 3369 material testing machine was used, the stretching velocity was $0.1 \mathrm{~mm} / \mathrm{s}$, and the size of the specimen is shown in Figure 3(a). A broken specimen is shown in Figure 3(b); in order to strengthen the clamping effect, spiral grooves were sculptured on the holding surfaces. The stressstrain curves of GH4169 are shown in Figure 3(c).

2.2. Validation of the Numerical Model. Due to the irregular shape of the blade and the corresponding cavity surfaces, it is difficult to accurately measure or calculate the contact responses. The proportion of the dynamic energy and total energy is introduced to validate the FEM model; when the energy proportion is smaller than $5 \%$, the results of the FEM model are validated. Figure 4(a) shows the variation of dynamic energy and total energy, and Figure 4(b) shows variation of the energy proportion between the dynamic energy and the total energy. The figures show that the total energy exceeds the dynamic energy when the simulation completed $1 \%$, and the energy proportion exceeds $5 \%$ when the simulation completed $1.5 \%$. Hence, the FEM model is valid, and the simulated results are credible.

\section{The Contact Responses and Evolutions of Roller Cavity Surfaces}

The load range, number of load cycles, and specimen geometry are three major variables for fatigue failure [21-23]; hence, studying the load distribution and evolution is significant for the moulding process. In the blade rolling process, the roller cavity surfaces squeeze the work blank with the rotation of rollers, and the blank occurred plastic deformation by the squeezing action. The rollers undergo squeezing and friction in the rolling process, and the contact responses of the roller cavity surfaces comprise three portions: the contact pressure which overcomes resistance from the blank's plastic deformation, the shear stress to overcome the friction resistance between the roller and the blank, and the local microslip between the roller and the blank. Hence, contact interfaces are constantly changing with the rotary mould, and the region and magnitude of the contact responses on cavity surfaces are changed constantly. An accurate quantitative distribution of the local contact pressure and local sliding over the rolling bite was determined, and the results are shown in Figure 5 [24]. According to Figure 5, the local contact pressure over the rolling bite demonstrates a hill profile, and the local sliding occurs at the forward slip and backward slip zones with the two peaks. Due to the complex shape of the blade, the contact responses in the 


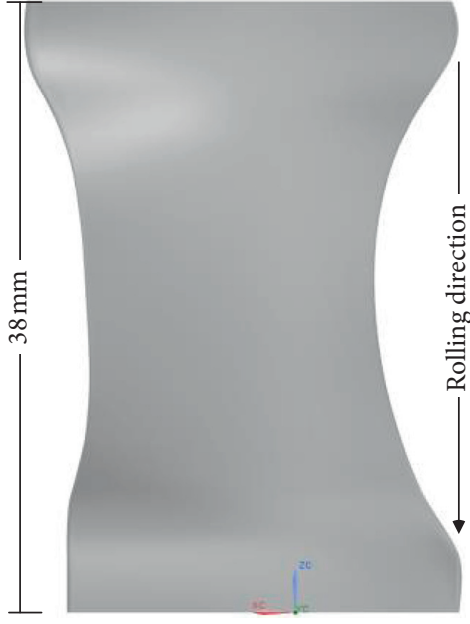

(a)

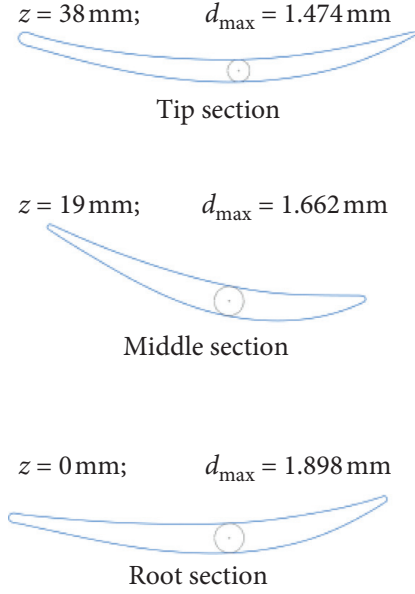

(b)

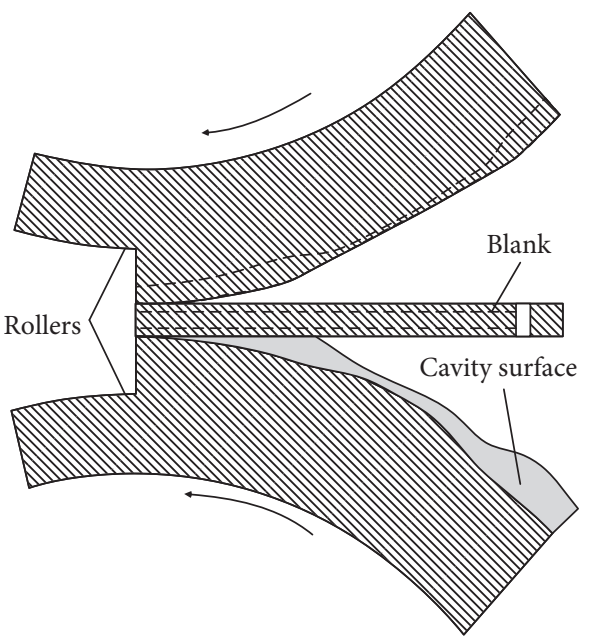

(c)

Figure 1: The process of the rolling blade: (a) CAD model of the blade, (b) the representative cross section, and (c) a schematic of the blade rolling process.

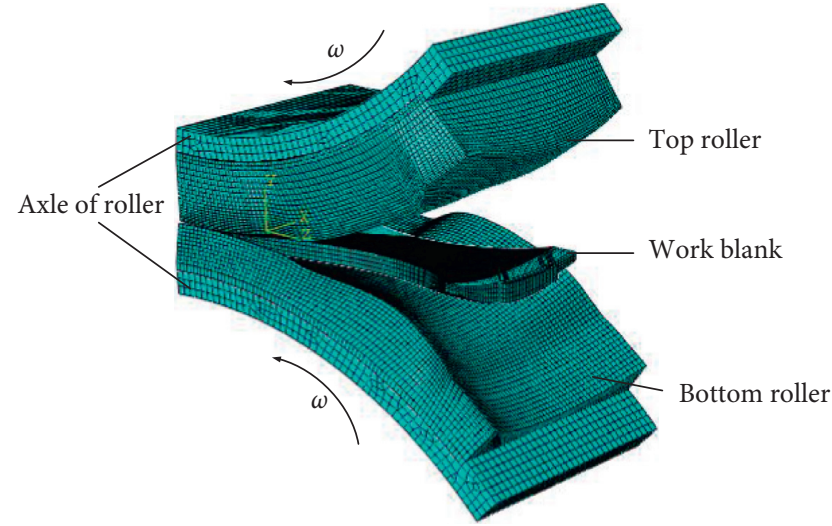

FIGURE 2: The FEM model for the blade rolling process.

blade rolling process are more intricate than those in the symmetric rolling process along the rolling direction.

\subsection{The Contact Pressure Distribution and Evolution of Roller} Cavity Surfaces. The plastic deformation resistance of the blank produces contact pressure at cavity surfaces. The contact interface and the magnitude of contact pressure are changed constantly in the blade rolling process. In order to investigate the time-dependent evolution of contact pressure, the transient magnitude of contact pressure over the roller cavities was recorded and plotted to present the contact pressure distribution. Meanwhile, the 6 uniform transients, contact pressure distribution of the roller cavities was enumerated to present the evolution of contact pressure. The contact pressure distribution and evolution over the cavity surfaces are shown in Figure 6.

If the total time for a blade rolling process is $T$, the contact interface is presented as a half ellipse at the transient of $T / 6$, shown in Figure 6(a). The rotary rollers and blank began to contact at the arc line and separate at the chord line. The maximum contact pressure over the top and bottom roller cavities is $1579.3 \mathrm{MPa}$ and $1469.7 \mathrm{MPa}$, respectively, the maximum contact over the top cavity is slightly bigger than that of the bottom cavity because of the blank bending and extruding toward the suction surface of the blade. The contact interface presented as trapezium at the transient of $2 T / 6$, shown in Figure 6(b); because the chord lengths of blade sections are shrunken, the roller cavities begin to contact with the blank at the top baseline and separate at the bottom baseline. The maximum contact pressure over the top and bottom roller cavities is 1648.2 MPa and 1649.1 MPa, respectively, and the higher contact pressure is present at the center of trapezium because of the tight twisting of blade sections which induced severe deformation resistance of the blank. The contact interfaces presented as hourglass-shaped at the transient of 3T/6 and 4T/6, shown in Figures 6(c) and 6(d). The maximum contact pressure over the top roller cavities is $1627.6 \mathrm{MPa}$ and $1648.7 \mathrm{MPa}$, and the maximum contact pressure over the bottom roller cavities is 1639.2 $\mathrm{MPa}$ and 1647.2 $\mathrm{MPa}$, respectively. The higher contact pressure is presented at glass bulbs of the hourglass because of the severe bending of blade sections which induced severe resistance of bending deformation. The contact interfaces presented as trapezium at the transient of 5T/6, shown in Figure 6(e); because the chord lengths of blade sections are expanded, the roller cavities begin to contact with the blank at the bottom baseline and separate at the top baseline. The maximum contact pressure over the top and bottom roller cavities is $1647.1 \mathrm{MPa}$ and $1620.8 \mathrm{MPa}$, respectively. The contact interfaces presented as a rectangle at the transient of $T$, shown in Figure 6(f). The maximum contact pressure over the top and bottom roller cavities is $1225.7 \mathrm{MPa}$ and $1186.9 \mathrm{MPa}$, respectively.

According to the results of the contact pressure distributions and evolutions over the cavity surfaces, the contact pressure distributions and evolutions are complex, and the contact interfaces and pressure distributions varied frequently. The maximum contact pressure is generally present at the center of the contact interface. 
TABLE 1: Material properties of work blank and roller.

\begin{tabular}{lccccc}
\hline & Material definitions & Density $\left(\mathrm{kg} / \mathrm{m}^{3}\right)$ & Elastic modulus $(\mathrm{GPa})$ & Poisson's ratio & Yield strength $(\mathrm{MPa})$ \\
\hline Work blank & Elastic-plastic & 8160 & 210 & 0.29 & 1200 \\
Roller & Elastic & 7700 & 235 & 0.3 & - \\
\hline
\end{tabular}

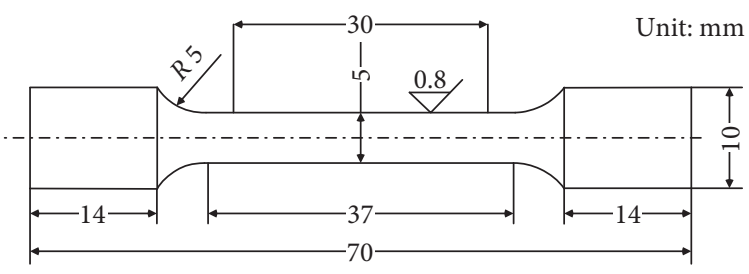

(a)

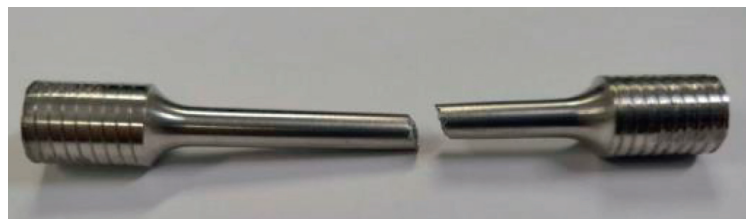

(b)

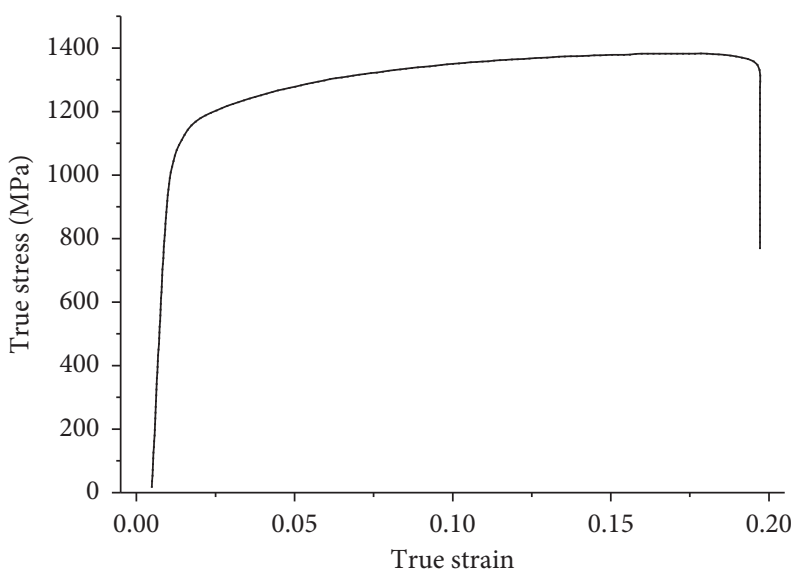

(c)

FIGURE 3: The material plasticity testing of GH4169: (a) the size of the specimen, (b) the broken specimen, and (c) the stress-strain curves of GH4169.

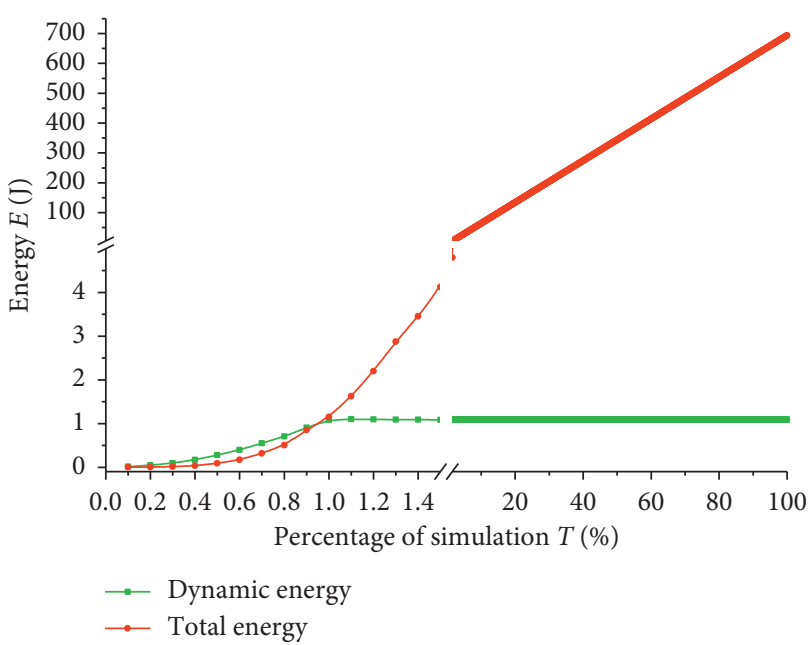

(a)

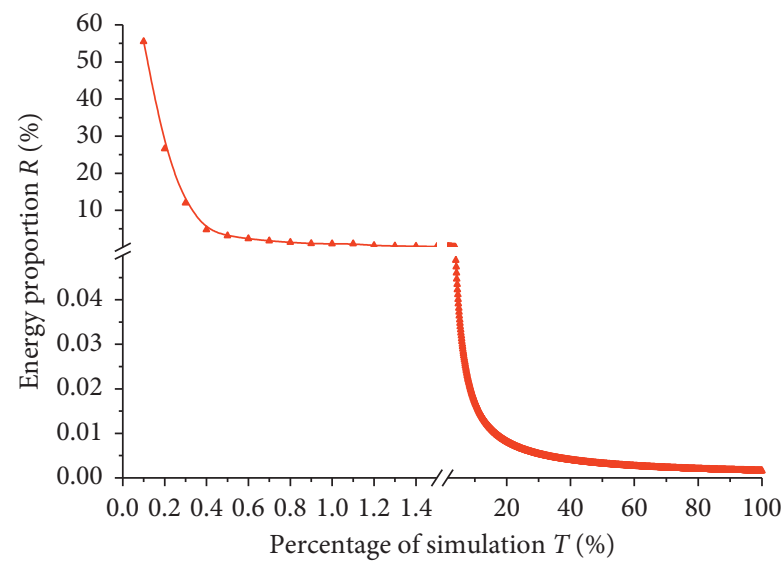

(b)

FIGURE 4: The energy analysis of the FEM model: (a) the variation of dynamic energy and total energy; (b) the variation of the energy proportion between the dynamic energy and the total energy. 


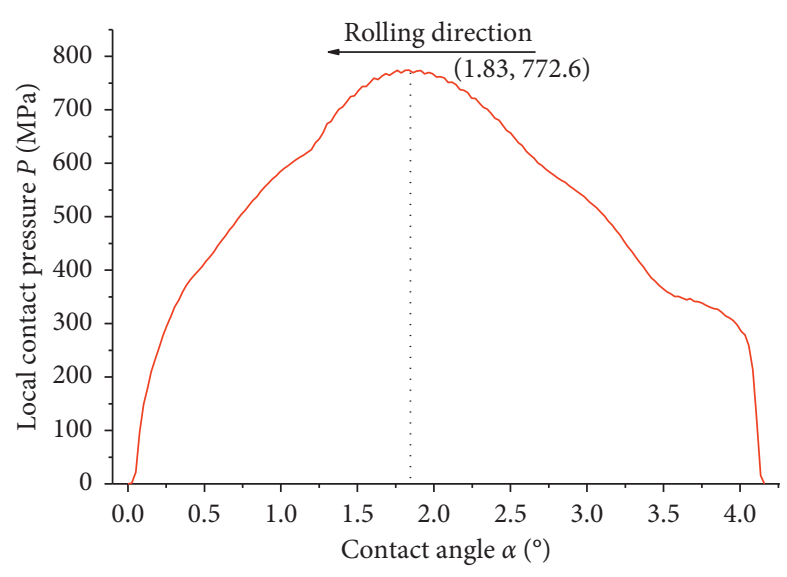

(a)

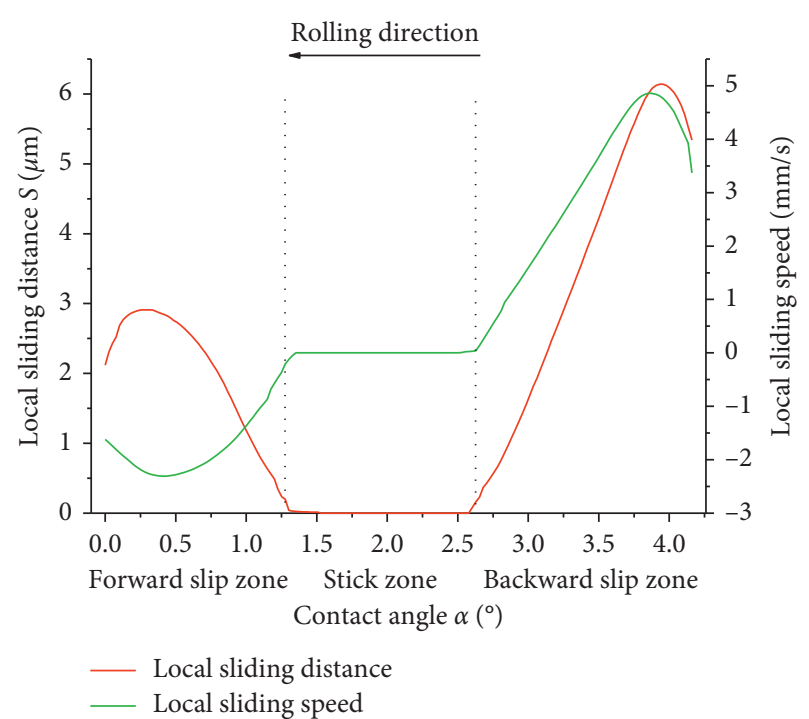

(b)

FIgURE 5: Distribution of local contact pressure (a) and local sliding (b) over the rolling bite [24].

In order to evaluate the pressure magnitude over roller cavities accurately, the pressure magnitude was an average value of the maximum contact pressure and four nearest nodes' contact pressure. The ratio between the standard deviation and the mean value is usually smaller than $3.82 \%$; hence, the tested results were valid. The evolution of contact pressure magnitudes and their standard deviations are presented in Figure 7. The pressure magnitudes and their evolution over the top and bottom cavities were similar. The pressure magnitudes were a little bit lower at the beginning of the rolling process, then remained nearly constant, and reduced obviously at the ending of the rolling process. The maximum thickness of the blank's section was uniform; however the maximum thickness of the blade's tip section was smaller than the maximum thickness of the blade's bottom section, shown in Figure 1(b). Hence, the thickness reduction of the blank decreased, and the magnitudes of contact pressure reduced at the ending of the rolling process.

3.2. The Shear Stress Distribution and Evolution of Roller Cavity Surfaces. In the rolling process, a gradually shrunken zone was shaped between a pair of conjugated rotary rollers, and the blank was screwed into the deformation zone by shear stress; hence, obtaining the distribution and evolution of the shear stress between the roller cavities and the blank is essential for investigating the wear of rollers. In order to investigate the time-dependent evolution of the shear stress, the transient magnitude of the shear stress over the roller cavities was recorded and plotted to present the shear stress distribution. Meanwhile, the 6 uniform transients' shear stress distribution of the roller cavities was enumerated to present the evolution of shear stress. The shear stress distribution and evolution over the cavity surfaces are shown in Figure 8.
The distribution of shear stress is shown in Figure 8(a) at the transient of $T / 6$. The contact interface was divided into two zones by a transition area which is perpendicular to the rolling direction. According to the previous research [24], the rolling contact interface can be divided into three parts along the rolling direction, namely, the backward slip zone, the stick zone, and the forward slip zone. The direction of the shear stress is the same to the rolling direction in the backward slip zone, and it is shown as blue zones in Figure 8 . The shear stress is almost zero in the transition area. The direction of the shear stress is adverse to the rolling direction in the forward slip zone, and it is shown as red zones in Figure 8 . When the blade rolling process evolves at the transient of $T / 6$, the maximum shear stress in the forward and backward slip zones of the top roller cavity is $310.4 \mathrm{MPa}$ and $428.3 \mathrm{MPa}$, respectively, and the maximum shear stress in the forward and backward slip zones of the bottom roller cavity is $301.9 \mathrm{MPa}$ and $413.8 \mathrm{MPa}$, respectively, shown in Figure $8(a)$. The maximum shear stress in the forward slip zone is smaller than that in the backward slip zone because the backward slip zone suffers severe friction which screwed the blank into the deformation zone by shear stress. When the blade rolling process evolves at the transient of $2 T / 6$, the maximum shear stress in the forward and backward slip zones of the top roller cavity is $300.8 \mathrm{MPa}$ and $414.1 \mathrm{MPa}$, respectively, and the maximum shear stress in the forward and backward slip zones of the bottom roller cavity is $292.1 \mathrm{MPa}$ and $405.8 \mathrm{MPa}$, respectively, shown in Figure $8(\mathrm{~b})$. When the blade rolling process evolves at the transient of $3 T / 6$, the maximum shear stress in the forward and backward slip zones of the top roller cavity is $291.8 \mathrm{MPa}$ and $406.9 \mathrm{MPa}$, respectively, and the maximum shear stress in the forward and backward slip zones of the bottom roller cavity is $289.4 \mathrm{MPa}$ and $406.1 \mathrm{MPa}$, respectively, shown in Figure $8(\mathrm{c})$. When the blade rolling process evolves at the transient of $4 T / 6$, the maximum shear stress in the forward 


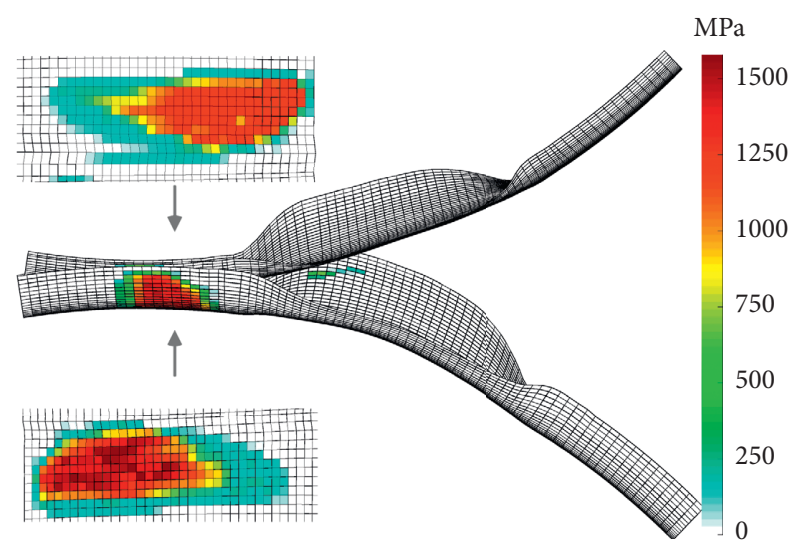

(a)

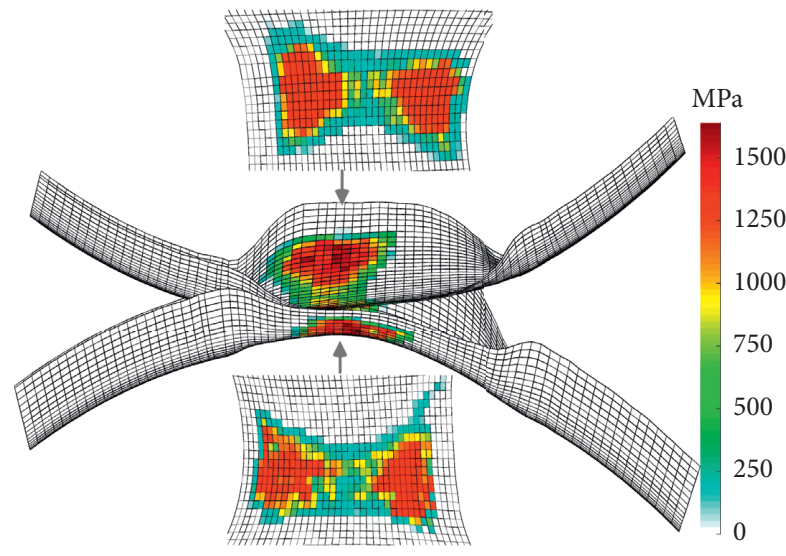

(c)

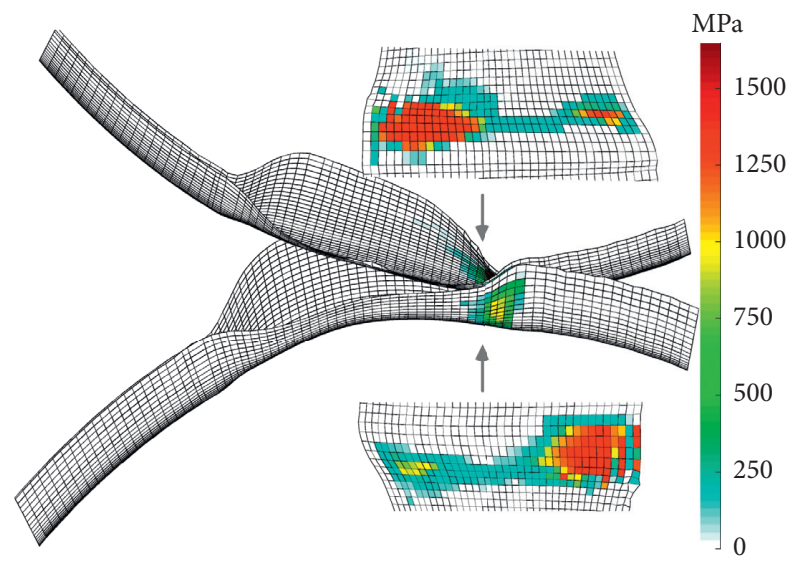

(e)

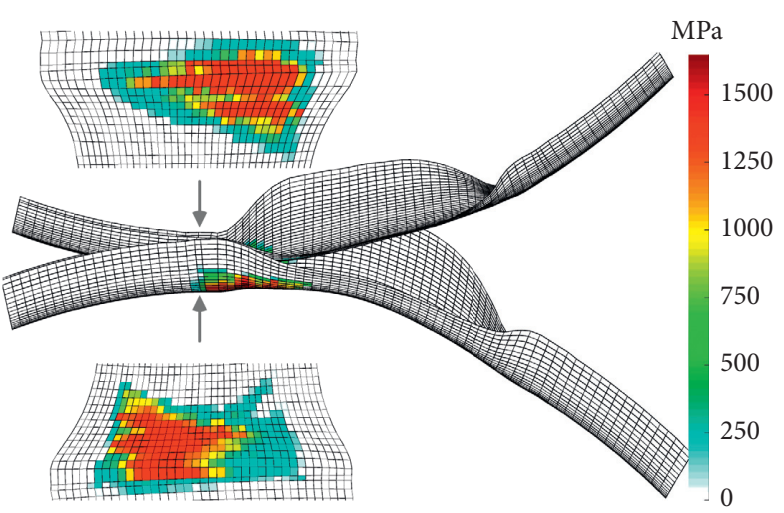

(b)

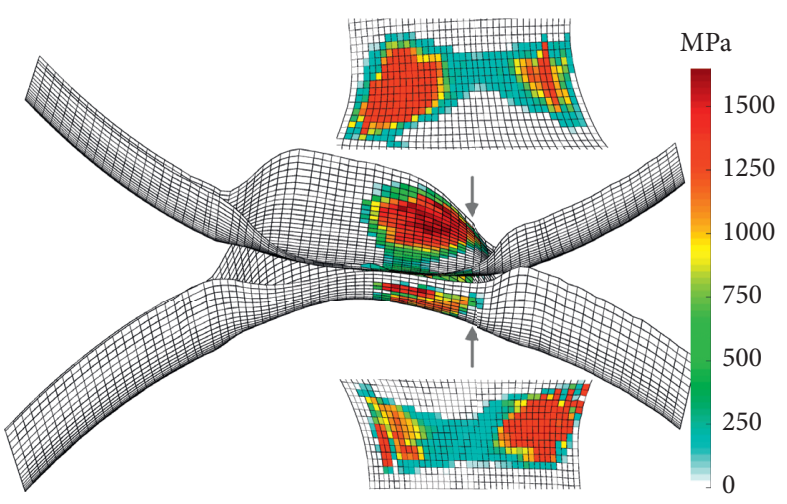

(d)

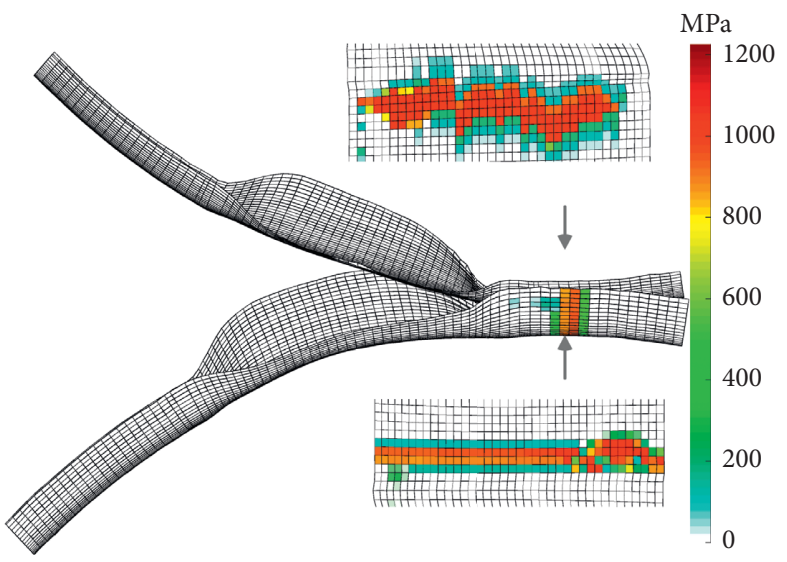

(f)

Figure 6: The transient contact pressure distribution at six different instances during the simulation: (a) T/6, (b) 2T/6, (c) $3 T / 6$, (d) 4T/6, (e) $5 T / 6$, and (f) $T$.

and backward slip zones of the top roller cavity is 279.5 $\mathrm{MPa}$ and $365.9 \mathrm{MPa}$, respectively, and the maximum shear stress in the forward and backward slip zones of the bottom roller cavity is $276.3 \mathrm{MPa}$ and $364.1 \mathrm{MPa}$, respectively, shown in Figure $8(\mathrm{~d})$. When the blade rolling process evolves at the transient of 5T/6, the maximum shear stress in the forward and backward slip zones of the top roller cavity is $241.9 \mathrm{MPa}$ and $307.8 \mathrm{MPa}$, respectively, and the maximum shear stress in the forward and backward slip zones of the bottom roller cavity is $244.1 \mathrm{MPa}$ and 296.1 MPa, respectively, shown in Figure 8(e). When the blade rolling process evolves at the transient of $T$, the maximum shear stress in the forward and backward slip zones of the top roller cavity is $192.3 \mathrm{MPa}$ and $255.0 \mathrm{MPa}$, respectively, and the maximum shear stress in the forward and backward slip zones of the bottom roller cavity is 169.2 $\mathrm{MPa}$ and 214.2 $\mathrm{MPa}$, respectively, shown in Figure 8(f). 


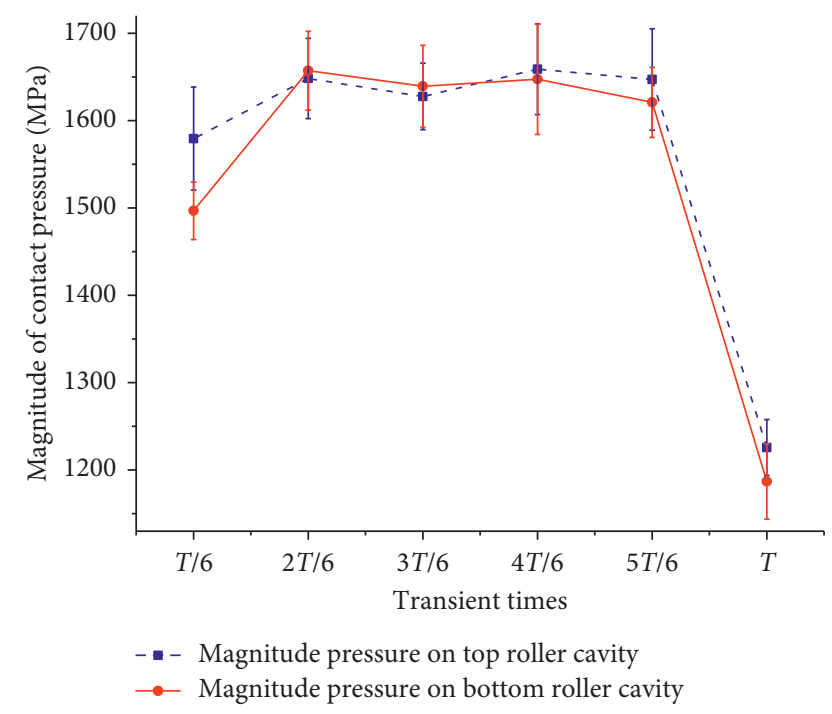

FIgURE 7: The evolution of the pressure magnitudes and their standard deviations over roller cavities.

According to the results of the shear stress distribution and evolution over the cavity surfaces, the rolling contact interface can be divided into the backward slip zone, the stick zone, and the forward slip zone. The stick zone was a narrow and invert belt perpendicular to the rolling direction, the stick zone was the boundary of the forward and backward slip zones, and the shear stress in the stick zone was nearly zero. The shear stress magnitudes in the forward slip zone were smaller than those in the backward slip zone at every transient. The directions of the shear stress were the same to the rolling direction in the backward slip zone, and the directions of the shear stress were adverse to the rolling direction in the forward slip zone. Because the blank was screwed into the deformation zone by friction, hence, the integral force of the shear stress in the backward slip zone not only surmounts the shear stress in the forward slip zone but also overcomes the resistance to plastic flow of the blank.

In order to evaluate the shear stress magnitude over roller cavities accurately, the shear stress magnitude was an average value of the maximum shear stress and four nearest nodes' shear stress. The ratio between the standard deviation and the mean value is usually smaller than $10.66 \%$; the errors were acceptable, and the tested results were valid. The evolution of shear stress magnitudes and their standard deviations are presented in Figure 9. The magnitudes of shear stress over the forward and backward slip zones decreased with evolution of the blade rolling process. The maximum thickness of the blade's tip section was smaller than the maximum thickness of the blade's bottom section, shown in Figure 1(b), the rolling direction was directed from the tip section of the blade to the root section, and the deformation and reduction rate of the blank decreased in the blade rolling process.

\subsection{The Local Sliding Distribution and Evolution of Roller} Cavity Surfaces. Local sliding occurs between the roller cavities and the blank, and the local sliding induces wear.
Hence, obtaining the distribution and evolution of the local sliding between the roller cavities and blank is essential for investigating the wear of rollers. In order to investigate the time-dependent evolution of the local sliding, the transient magnitude of the local sliding velocity over the roller cavities was recorded and plotted to present the local sliding distribution. Meanwhile, the 6 uniform transients' local sliding velocity distribution of the roller cavities was enumerated to present the evolution of local sliding. The local sliding distribution and evolution over the cavity surfaces are shown in Figure 10.

The distribution of local sliding velocity is shown in Figure 10(a) at the transient of T/6. It is the same as the distribution of the shear stress, and the distribution was divided into two zones by a transition area which is perpendicular to the rolling direction. The direction of the local sliding is the same to the rolling direction in the backward slip zone, and it is shown as blue zones in Figure 7. The local sliding is almost zero in the transition area. The direction of the local sliding is adverse to the rolling direction in the forward slip zone, and it is shown as red zones in Figure 10. When the blade rolling process evolves at the transient of $T /$ 6 , the magnitude of local sliding velocity in the forward and backward slip zones of the top roller cavity is $2.99 \mathrm{~mm} / \mathrm{s}$ and $7.52 \mathrm{~mm} / \mathrm{s}$, respectively, and the magnitude of local sliding velocity in the forward and backward slip zones of the bottom roller cavity is $2.77 \mathrm{~mm} / \mathrm{s}$ and $7.96 \mathrm{~mm} / \mathrm{s}$, respectively, shown in Figure 10(a). The magnitude of local sliding velocity in the forward slip zone is smaller than that in the backward slip zone, and the proportion of the backward slip zone is normally bigger than that of the forward slip zone [24]. When the blade rolling process evolves at the transient of $2 T / 6$, the magnitude of local sliding velocity in the forward and backward slip zones of the top roller cavity is $4.24 \mathrm{~mm} / \mathrm{s}$ and $6.45 \mathrm{~mm} / \mathrm{s}$, respectively, and the magnitude of local sliding velocity in the forward and backward slip zones of the bottom roller cavity is $4.70 \mathrm{~mm} / \mathrm{s}$ and $9.06 \mathrm{~mm} /$ $\mathrm{s}$, respectively, shown in Figure 10(b). When the blade rolling process evolves at the transient of $3 T / 6$, the magnitude of local sliding velocity in the forward and backward slip zones of the top roller cavity is $5.06 \mathrm{~mm} / \mathrm{s}$ and $6.30 \mathrm{~mm} /$ $\mathrm{s}$, respectively, and the magnitude of local sliding velocity in the forward and backward slip zones of the bottom roller cavity is $3.36 \mathrm{~mm} / \mathrm{s}$ and $9.75 \mathrm{~mm} / \mathrm{s}$, respectively, shown in Figure 10(c). When the blade rolling process evolves at the transient of $4 T / 6$, the magnitude of local sliding velocity in the forward and backward slip zones of the top roller cavity is $6.55 \mathrm{~mm} / \mathrm{s}$ and $7.26 \mathrm{~mm} / \mathrm{s}$, respectively, and the magnitude of local sliding velocity in the forward and backward slip zones of the bottom roller cavity is $6.40 \mathrm{~mm} / \mathrm{s}$ and $9.57 \mathrm{~mm} /$ $\mathrm{s}$, respectively, shown in Figure 10(d). When the blade rolling process evolves at the transient of $5 T / 6$, the magnitude of local sliding velocity in the forward and backward slip zones of the top roller cavity is $2.73 \mathrm{~mm} / \mathrm{s}$ and $3.30 \mathrm{~mm} /$ $\mathrm{s}$, respectively, and the magnitude of local sliding velocity in the forward and backward slip zones of the bottom roller cavity is $2.74 \mathrm{~mm} / \mathrm{s}$ and $3.50 \mathrm{~mm} / \mathrm{s}$, respectively, shown in Figure 10(e). When the blade rolling process evolves at the transient of $T$, the magnitude of local sliding velocity in the 


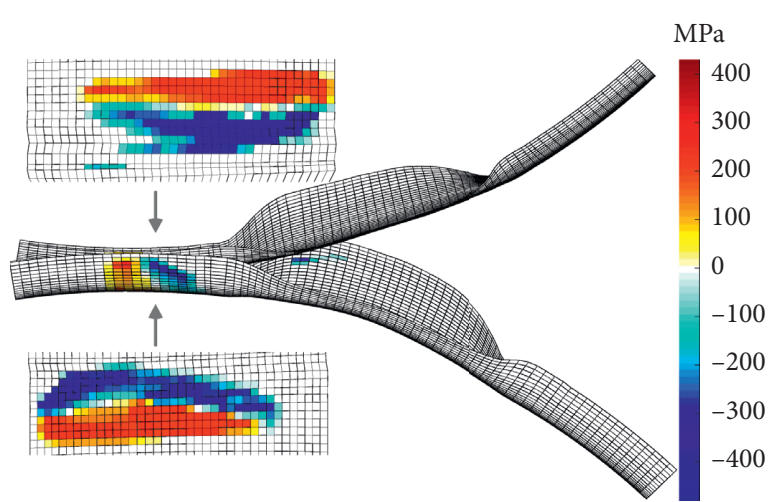

(a)

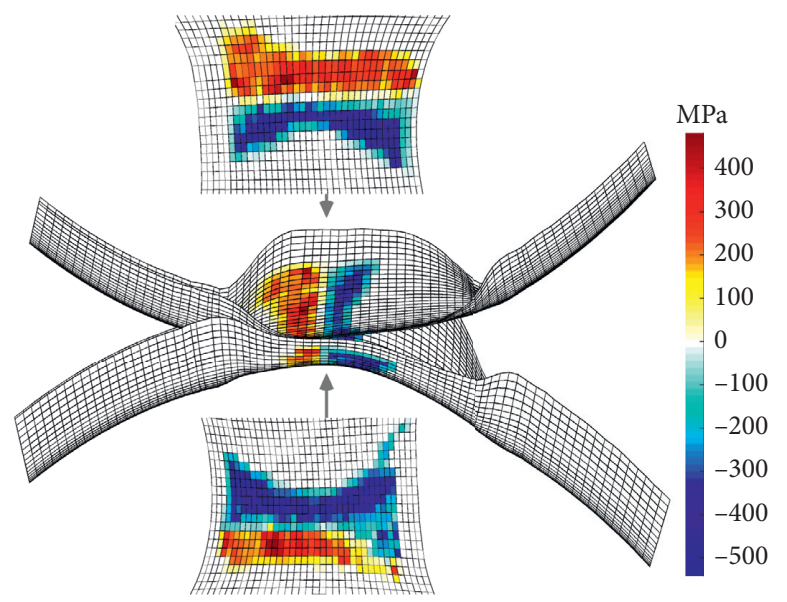

(c)

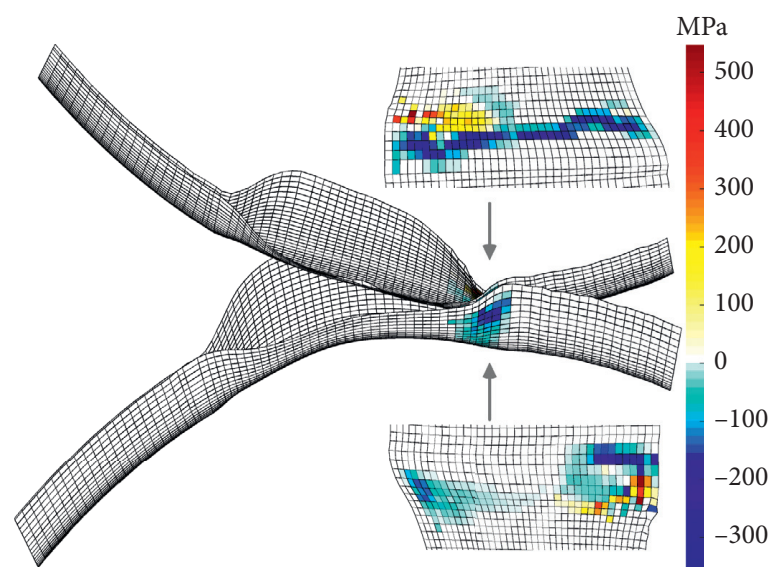

(e)

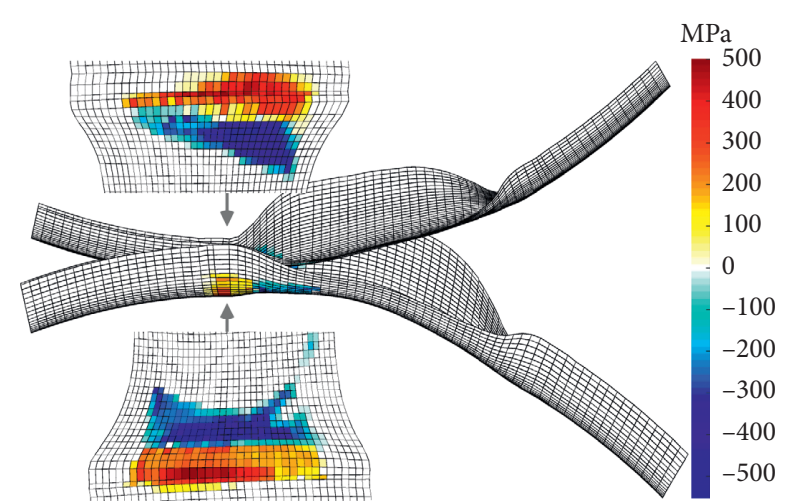

(b)

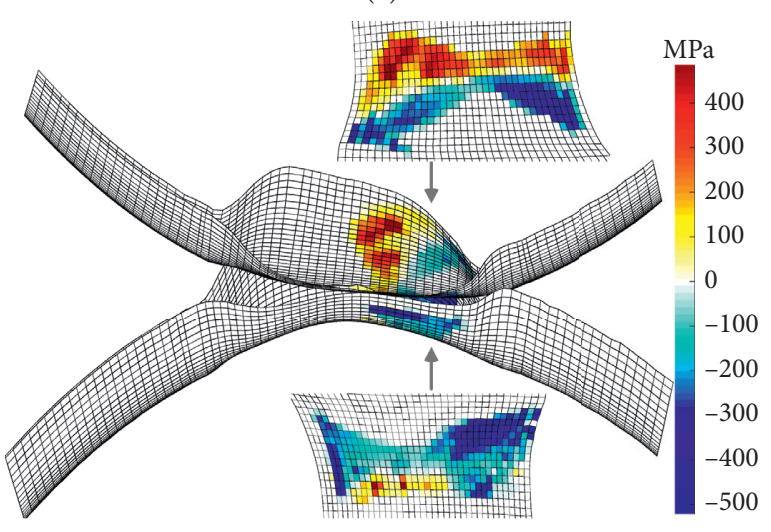

(d)

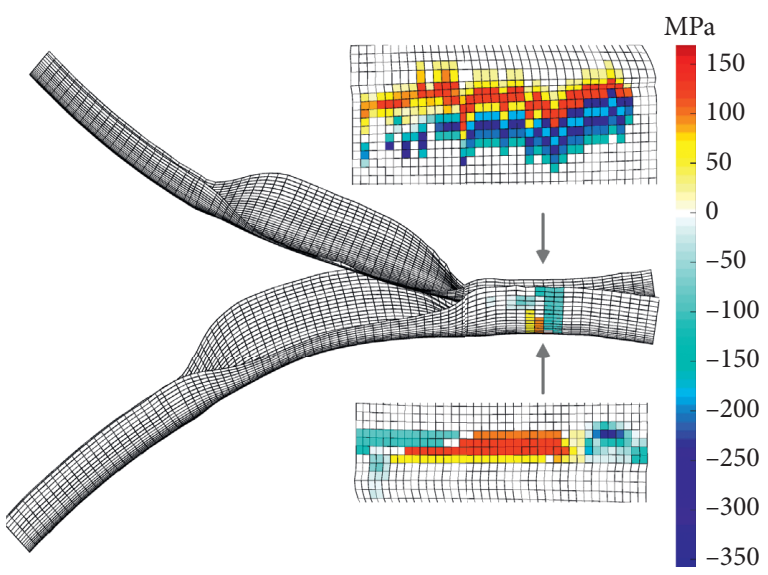

(f)

FIGURE 8: The transient shear stress distribution at six different instances during the simulation: (a) T/6, (b) 2T/6, (c) 3T/6, (d) 4T/6, (e) 5T/6, and (f) $T$.

forward and backward slip zones of the top roller cavity is $1.08 \mathrm{~mm} / \mathrm{s}$ and $1.47 \mathrm{~mm} / \mathrm{s}$, respectively, and the magnitude of local sliding velocity in the forward and backward slip zones of the bottom roller cavity is $0.71 \mathrm{~mm} / \mathrm{s}$ and $1.41 \mathrm{~mm} /$ s, respectively, shown in Figure 10(f).

According to the results of the local sliding velocity distribution and evolution over the cavity surfaces, the local sliding distribution was similar to the shear stress distribution, namely, the rolling contact interface was divided into the backward slip zone, the stick zone, and the forward slip zone. The stick zone was a narrow and invert belt perpendicular to the rolling direction, and the stick zone was the boundary of the forward and backward slip zones. The direction of the local sliding was the same to the rolling direction in the backward slip zone, and the direction of the local sliding was adverse to the rolling direction in the forward slip zone. The amplitude distributions of local sliding were in a $\mathrm{V}$-shape, the local sliding in the stick zone 


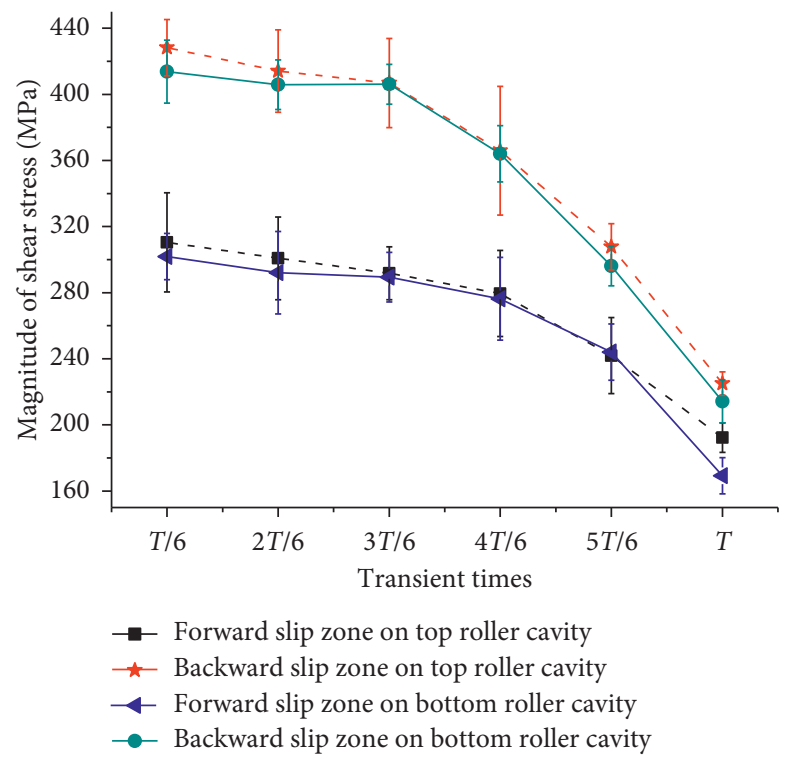

FIgURE 9: The evolution of the shear stress magnitudes and their standard deviations over roller cavities.

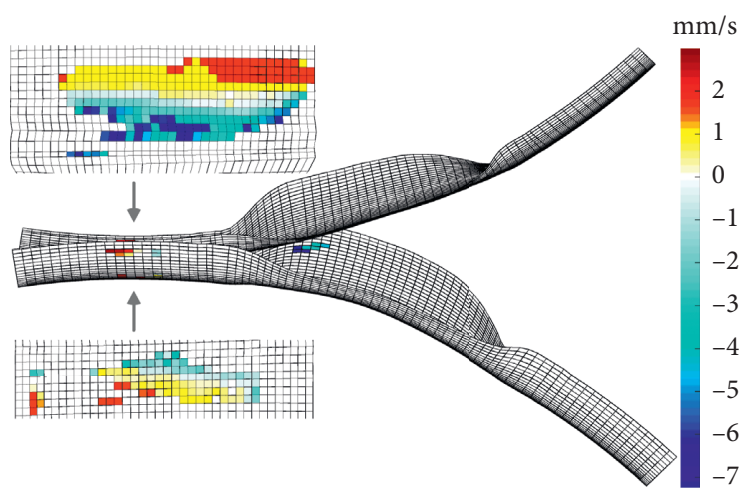

(a)

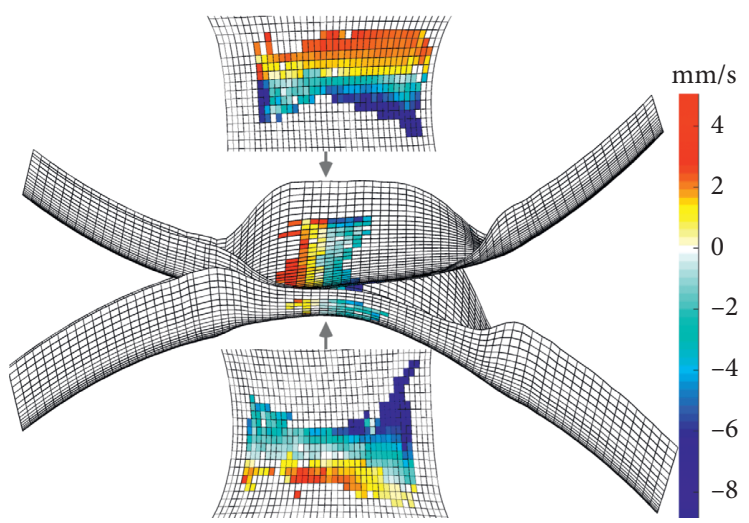

(c)

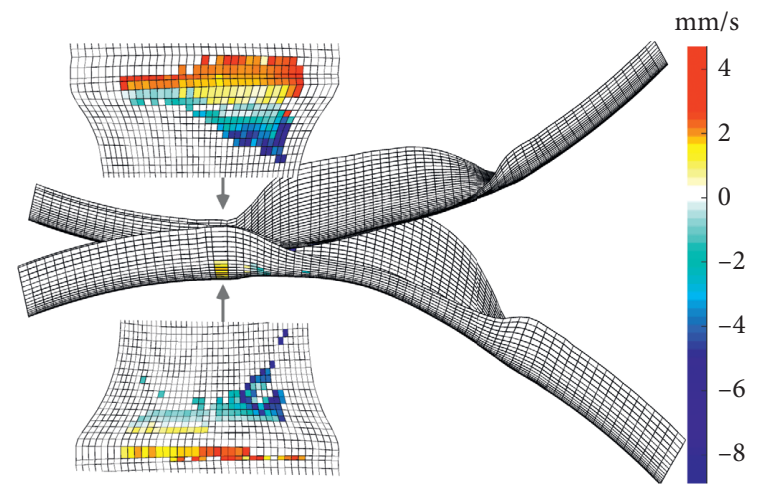

(b)

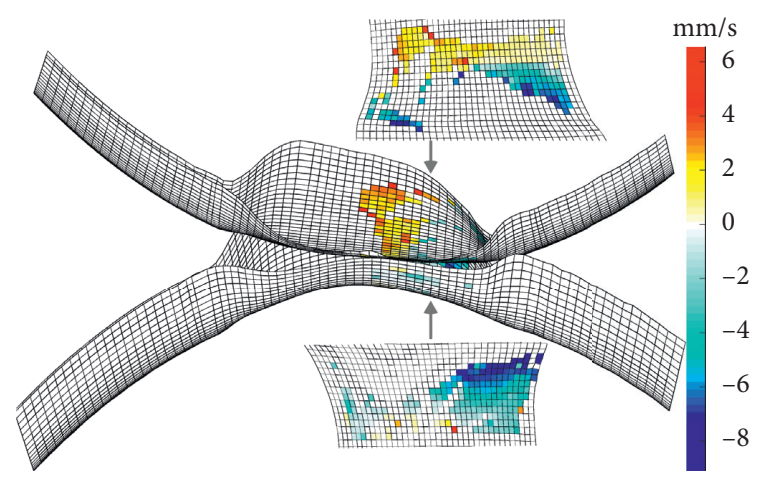

(d)

Figure 10: Continued. 


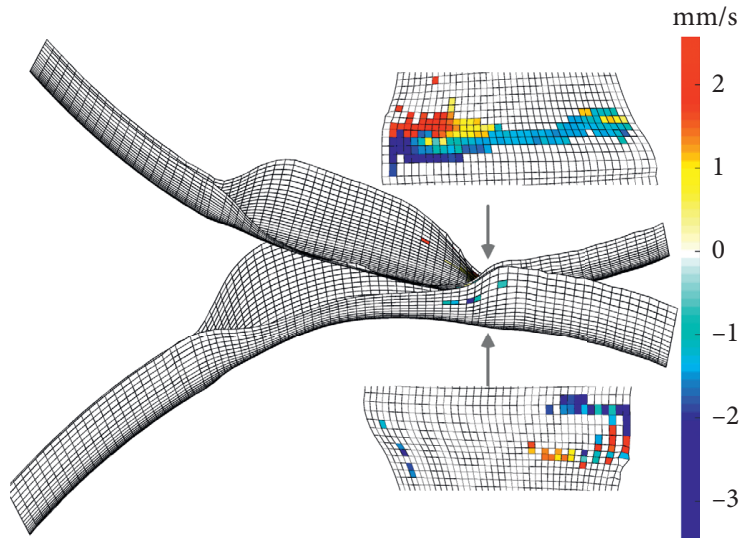

(e)

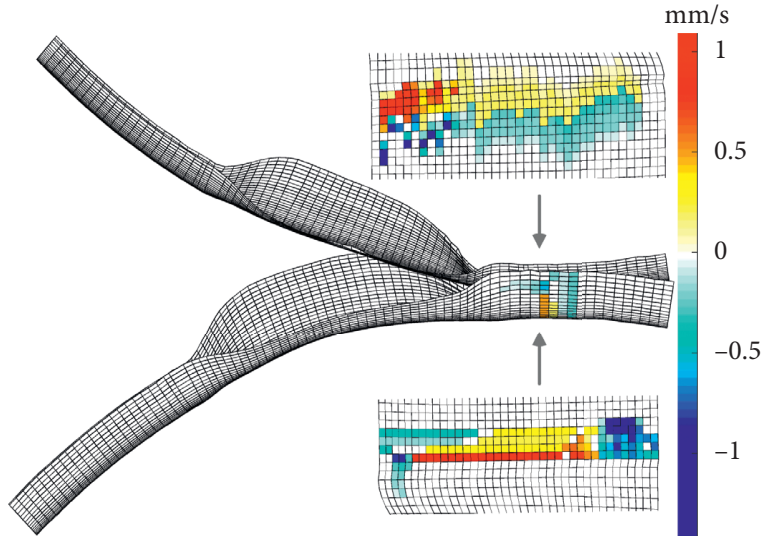

(f)

Figure 10: The transient local sliding at six different instances during the simulation: (a) T/6, (b) $2 T / 6$, (c) $3 T / 6$, (d) 4T/6, (e) 5T/6, and (f) T.

was nearly zero, the bigger sliding in backward and forward slip zones was present at the boundaries of rolling entrance and exit sections. The magnitudes of local sliding at the rolling entrance sections were bigger than those at the rolling exit sections in every transient.

In order to evaluate the local slip magnitude over roller cavities accurately, the local slip magnitude was an average value of the maximum local slip and four nearest nodes' local slip. The ratio between the standard deviation and the mean value is usually smaller than $16.05 \%$; the errors were acceptable, and the tested results were valid. The evolution of local sliding magnitudes and their standard deviations are presented in Figure 11. In general, the local sliding velocity magnitudes increased firstly and decreased sharply at $2 T / 3$. The local sliding velocity magnitudes at the backward slip zones were always bigger than those at the forward slip zones. The maximum thickness of the blade's tip section was smaller than the maximum thickness of the blade's bottom section, shown in Figure 1(b), the rolling direction was directed from the tip section of the blade to the root section, the reduction rate of the blank decreased in the blade rolling process, and the rolling bites shrank.

\section{Conclusions}

This paper investigated the contact response distributions and evolutions of the roller cavities in the compressor blade rolling process based on the FEM. It was the first study to quantify the distributions and evolutions of contact responses for rolling irregular components. It was the essential step for studying the wear of rollers. The major findings are summarized as follows:

(1) The contact between the roller and the blade was time-location-dependent evolution in the blade rolling process. The contact interface was constantly updated and replaced with the rotation of the roller. The quantized contact pressure distribution was obtained, and the evolution of pressure distribution varied frequently. The maximum contact pressure is

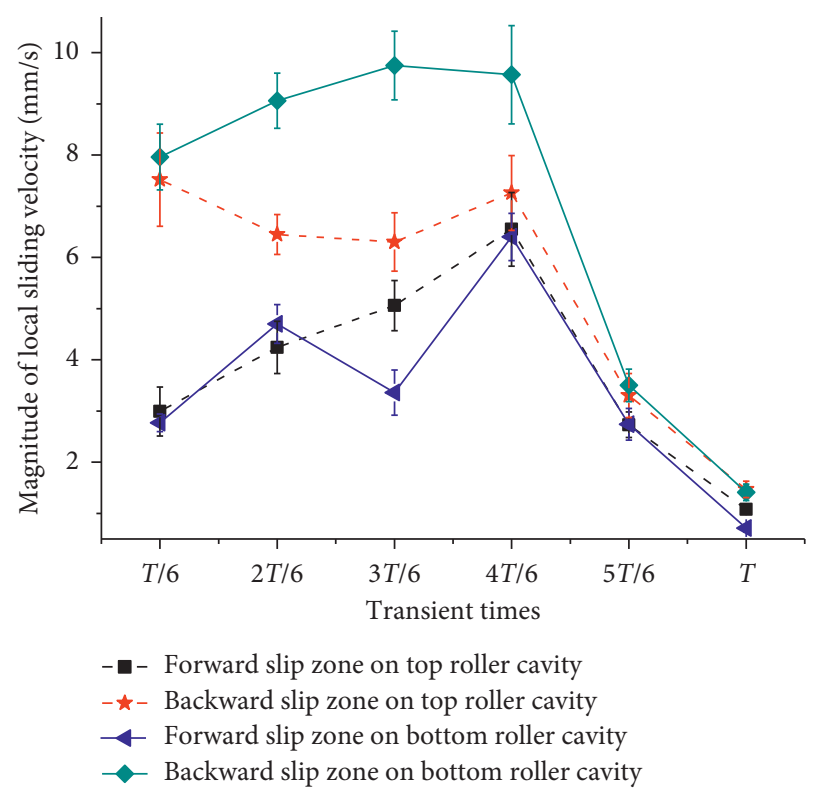

FIgURE 11: The evolution of the local slip magnitudes and their standard deviations over roller cavities.

generally present at the center of the contact interface at every transient.

(2) The rolling contact interface can be divided into the backward slip zone, the stick zone, and the forward slip zone based on the shear stress distribution. The shear stress magnitudes in the forward slip zone were smaller than those in the backward slip zone at every transient. The directions of the shear stress were the same to the rolling direction in the backward slip zone, and the directions of the shear stress were adverse to the rolling direction in the forward slip zone.

(3) The amplitude distributions of local sliding were in a $\mathrm{V}$-shape, the local sliding in the stick zone was nearly zero, and the bigger sliding in backward and forward slip zones was present at the boundaries of rolling 
entrance and exit sections. The magnitudes of local sliding at the rolling entrance sections were bigger than those at the rolling exit sections in every transient. The directions of the local sliding were the same to the rolling direction in the backward slip zone, and the directions of the local sliding were adverse to the rolling direction in the forward slip zone.

\section{Data Availability}

The data used to support the findings of this study are available from the corresponding author upon request.

\section{Conflicts of Interest}

The authors declare no conflicts of interest regarding the publication of this paper.

\section{Authors' Contributions}

Qichao Jin designed and conducted the research and wrote the manuscript, Wenhu Wang discussed the research and rechecked the results, and Ruisong Jiang discussed the research and revised the manuscript.

\section{Acknowledgments}

This work was supported by the Fundamental Research Funds for the Central Universities, CHD (nos. 300102250102 and 300102250202).

\section{References}

[1] C. Ren, J. Chen, H. Pan, X. Huang, and H. Zhu, "Wear mechanism of PA6 roller used in cold rolling process," Engineering Failure Analysis, vol. 28, pp. 311-317, 2013.

[2] Q. Jin, W. Wang, W. Yan, and R. Jiang, "Springback and forward slip compensation in designing roller cavity surfaces for net-shape rolling compressor blades," Materials and Manufacturing Processes, vol. 32, no. 12, pp. 1442-1449, 2017.

[3] Z. Cui, S. Bhattacharya, D. E. Green, and A. T. Alpas, "Mechanisms of die wear and wear-induced damage at the trimmed edge of high strength steel sheets," Wear, vol. 426427, pp. 1635-1645, 2019.

[4] L. C. Brezeanu, "Contact stresses: analysis by finite element method (FEM)," Procedia Technology, vol. 12, pp. 401-410, 2014.

[5] L. C. Brezeanu, "Contact stresses between two cylindrical bodies-cylinder and cylindrical cavity with parallel axes-part II: 2D and 3D FEA comparative study," Procedia Technology, vol. 19, pp. 177-184, 2015.

[6] X. Dong, X. Yin, Q. Deng et al., "Local contact behavior between elastic and elastic-plastic bodies," International Journal of Solids and Structures, vol. 150, pp. 22-39, 2018.

[7] K. J. Jobin, M. N. Abhilash, and H. Murthy, "A simplified analysis of $2 \mathrm{~d}$ sliding frictional contact between rigid indenters and FGM coated substrates," Tribology International, vol. 108, pp. 174-185, 2017.

[8] G.-N. Chu, G. Chen, C.-Y. Lin, Z.-G. Fan, and H. Li, “Analytical model for tube hydro-forging: prediction of die closing force, wall thickness and contact stress," Journal of
Materials Processing Technology, vol. 275, Article ID 116310, 2020.

[9] D. Weisz-Patrault, A. Ehrlacher, and N. Legrand, "Analytical inverse solution for coupled thermoelastic problem for the evaluation of contact stress during steel strip rolling," Applied Mathematical Modelling, vol. 37, no. 4, pp. 2212-2229, 2013.

[10] D. Weisz-Patrault, A. Ehrlacher, and N. Legrand, "Evaluation of contact stress during rolling process, by three dimensional analytical inverse method," International Journal of Solids and Structures, vol. 50, no. 20-21, pp. 3319-3331, 2013.

[11] D. Weisz-Patrault, L. Maurin, N. Legrand, A. Ben Salem, and A. Ait Bengrir, "Experimental evaluation of contact stress during cold rolling process with optical fiber Bragg gratings sensors measurements and fast inverse method," Journal of Materials Processing Technology, vol. 223, pp. 105-123, 2015.

[12] H. Y. Lee, J. H. Noh, and B. B. Hwang, "Surface stresses and flow modes on contact surface in a combined double cup extrusion process," Tribology International, vol. 64, pp. 215224, 2013

[13] W. A. Walbran, B. Verleye, S. Bickerton, and P. A. Kelly, "Prediction and experimental verification of normal stress distributions on mould tools during liquid composite moulding," Composites Part A: Applied Science and Manufacturing, vol. 43, no. 1, pp. 138-149, 2012.

[14] M. Sedighi and M. Mahmoodi, "An approach to simulate cold roll-forging of turbo-engine thin compressor blade," Aircraft Engineering and Aerospace Technology, vol. 81, no. 3, pp. 191-198, 2009.

[15] M. Sedighi and M. Mahmoodi, "Pressure distribution in cold rolling of turbo-engine thin compressor blades," Materials and Manufacturing Processes, vol. 27, no. 4, pp. 401-405, 2012.

[16] W. Y. Yan, M. P. Pereira, and B. F. Rolfe, "Tool wear in sheet metal stamping," Advanced Materials Research, vol. 421, pp. 750-753, 2011.

[17] W. Y. Yan, M. P. Pereira, and B. F. Rolfe, "Contact pressure evolution and its relation to wear in sheet metal forming," Wear, vol. 265, no. 11-12, pp. 1687-1699, 2008.

[18] W. Y. Yan, M. P. Pereira, and B. F. Rolfe, "Sliding distance, contact pressure and wear in sheet metal stamping," Wear, vol. 268, no. 11-12, pp. 1275-1284, 2010.

[19] M. P. Pereira, W. Yan, and B. F. Rolfe, "Wear at the die radius in sheet metal stamping," Wear, vol. 274, no. 3, pp. 355-367, 2012.

[20] A. Jurkiewicz, M. Pawlikowski, and Y. Pyryev, "Analytical, numerical and experimental analysis of the relationship between two rollers axes distance and the contact zone," International Journal of Mechanical Sciences, vol. 131-132, pp. 722-727, 2017.

[21] G. Qian and W.-S. Lei, "A statistical model of fatigue failure incorporating effects of specimen size and load amplitude on fatigue life," Philosophical Magazine, vol. 99, no. 17, pp. 2089-2125, 2019.

[22] G. Qian, C. Zhou, and Y. Hong, "Experimental and theoretical investigation of environmental media on very-high-cycle fatigue behavior for a structural steel," Acta Materialia, vol. 59, no. 4, pp. 1321-1327, 2011.

[23] S. Chen, Y. Wang, K. Tadaki, N. Hashimoto, and S. Ohnuki, "Suppression effect of nano-sized oxide particles on helium irradiation hardening in F82H-ODS steel," Journal of Nuclear Materials, vol. 455, no. 1-3, pp. 301-305, 2014.

[24] Q. Jin, W. Wang, R. Jiang, L. Chiu, D. Liu, and W. Yan, "A numerical study on contact condition and wear of roller in cold rolling," Metals, vol. 7, no. 9, p. 376, 2017. 